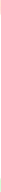

Artículo de Investigación - Proyecto PROMEP 2015 UASLP-222: “Planeación urbana centrada en la Persona. Un estudio crítico prospectivo de la planeación urbana en San Luis Potosí”. Recibido: 28-02-2017. Aprobado versión final: 15-02-2018

JEL: A13, A14, D60, D63, I31. * Pp. 195-213

\title{
Hacia una agenda urbana 2030 para América Latina. Planeación Urbana centrada en la Persona.
}

\author{
Towards a Urban Agenda 2030 for Latin America \\ Person-Centered Urban Planning
}

Benjamín Alva Fuentes - Marcela López Mares

MÉJICO

Resumen: Cada vez es más recurrente la frase "Hoy vivimos en un mundo urbano", haciendo referencia a que más de la mitad de la población (54\%) vive en ciudades; América Latina y el Caribe es la región más urbanizada (80 \%). Como resultado, las ciudades enfrentan grandes retos, derivados de su acelerado crecimiento. Con la finalidad de atender los principales problemas urbanos, la ONU definió en el objetivo 11 de Desarrollo Sostenible (ODS) las características de las ciudades hacia el 2030, para ello se diseñó una Agenda Urbana; sin embargo, ¿Cuáles deberían ser las bases para la implementación de esta Agenda en América Latina? El propósito de este documento es presentar algunas premisas para implementar la agenda urbana en la región, bajo un esquema centrado en las personas y la innovación.

Palabras clave: agenda urbana; ciudades; desarrollo urbano.

Abstract: The phrase "Today we live in an urban world" is increasingly recurrent, given that more than half of the population (54\%) lives in cities; Latin America and the Caribbean being the most urbanized region (80\%). As a result, cities face major challenges resulting from an accelerated urban growth. In order to address the most relevant urban problems, goal 11 of the UN's Urban Agenda defines the characteristics of cities towards the year 2030. However, on what bases should this agenda be implemented in Latin America? The purpose of this document is to present some premises for the implementation of the urban agenda in the region, under a scheme focused on people and innovation.

Keywords: urban agenda, cities, urban development.

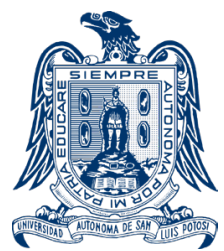

Benjamín Alva es Licenciado en Planeación Urbana por la Universidad Autónoma del Estado de México, Maestro en Estudios Urbanos por el Colegio de México y doctorante en Geografía por la Universidad Nacional Autónoma de México. Diplomado en Elementos Básicos de Gestión Estratégica para el Desarrollo Local y Regional por el ILPES/CEPAL, Chile. Profesor investigador de tiempo completo en la carrera de Diseño Urbano y del Paisaje y coordinador de investigación de la Facultad del Hábitat de la Universidad Autónoma de San Luis Potosí.

Contacto: balvaf@gmail.com 


\title{
Para uma Agenda Urbana 2030 para a América Latina
}

Planejamento urbano projetado para pessoas.

\begin{abstract}
Resumo: É cada vez mais a frase recorrente «Hoje vivemos em um mundo urbano» referindo-se a mais de metade da população (54\%) vive em cidades, na América Latina e no Caribe é a região mais urbanizada (80\%); No entanto, as cidades enfrentam grandes desafios decorrentes do seu rápido crescimento. A fim de resolver os principais problemas urbanos, a ONU definiu o alvo 11 características desenvolvimento sustentável das cidades, em 2030, para uma agenda urbana foi projetado; Entretanto, ¿o que deve ser a base para a execução dessa agenda na América Latina? O objetivo deste documento é apresentar algumas premissas para a agenda urbana na região, de acordo com um esquema centrado nas pessoas e inovação.
\end{abstract}

Palavras-chave: Agenda urbana, ciudades, desenvolvimento urbano.

\section{Introducción}

$\mathrm{E}$

ste siglo representa un cambio en la forma de entender el fenómeno urbano, derivado de su alta complejidad y el impacto de nuevos procesos como la globalización, el uso intensivo del conocimiento y de las Tecnologías de la Información y la Comunicación (TIC). Académicos reconocidos como Saskia Sassen (1999), Paul Krugman (2004) y Joseph Stiglitz (2015) han estudiado la relación entre estos procesos y la reorganización del espacio; si bien no existe consenso sobre sus efectos positivos o negativos, es ampliamente aceptada la idea de que son fenómenos que han condicionado nuevas relaciones espaciales. La influencia de estos nuevos procesos y sus dinámicas espaciales asociadas han redefinido las agendas globales, como es el caso de los Objetivos del Milenio que dieron lugar a los Objetivos de Desarrollo Sostenible (ODS).

Los ODS son un conjunto de 17 propósitos y 169 metas para el año 2030, que deberán atender todos los países con la finalidad de erradicar la pobreza, reducir la desigualdad y luchar contra el cambio climático. Particularmente, el objetivo 11 incluye 10 metas que las ciudades deben alcanzar para ser más seguras, resilientes, sustentables e inclusivas; para ello se propone un enfoque que privilegia a las personas.

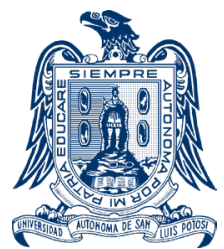

Marcela López es arquitecta por la Universidad Autónoma de San Luis Potosí, Maestra en Urbanismo y Ordenamiento Territorial, y doctora en Planeación Urbana por la Universidad de Chicago. Profesora de la carrera de Arquitectura en la UASLP y asistente de investigación en el Colegio de San Luis. Forma parte de los cuerpos académicos "Agua y Sociedad" y "Hábitat y Sustentabilidad del Territorio".

Contacto.marcela.lopez.mares@gmail.com 
Derivado de este objetivo 11, en 2016 se llevó a cabo la Conferencia Hábitat III sobre Vivienda y Desarrollo Urbano Sostenible, se acordó una Nueva Agenda Urbana (NAU), que subraya la importancia de la urbanización en la transformación de la población, de actividades económicas y de la sostenibilidad. En 2017 se elaboró el Plan de Acción Regional (PAR) para implementar la NAU en América Latina y el Caribe (ALC), que representa un importante avance, pero requiere herramientas adicionales como metodologías para acelerar la implementación.

De esta manera, un elemento que caracteriza los nuevos procesos son las relaciones en diferentes dimensiones, desde físicas hasta virtuales y en diversas escalas son un elemento condicionante en la implementación del PAR, de acuerdo con las particularidades de cada ciudad. A partir de lo anterior, el propósito de este documento es proponer un modelo de planeación y diseño urbano centrado en las personas, la innovación y la generación de valor, útil para los ODS, la NAU y el PAR.

Este documento se divide en tres apartados, el primero, a manera de diagnóstico, describe las grandes tendencias urbanas en ALC; en el segundo se hace una reflexión sobre el concepto de "la persona" así como de su importancia para la planeación, con la finalidad de abonar en las implicaciones de la NAU, además, por tratarse de una agenda global y su importancia para la región, se discute sobre la aplicación de visiones que integren la ciudad a escala global; $y$ en el tercero, a manera de resultado, se propone un modelo de planeación y diseño urbano centrado en las personas, útil en la implementación de la NAU y el PAR; se considera parte de la propuesta de Jan Gehl (2014) sobre la escala humana, principios de sociología urbana y de infraestructura del paisaje, se sintetiza una propuesta analítica con tres componentes: accesibilidad, conectividad y generación de valor; a manera de síntesis, se plantean algunas reflexiones para llevar a cabo este tipo de modelos de planeación en ciudades latinoamericanas. Este artículo parte de la premisa que, si la NAU plantea una nueva forma de hacer las cosas, no se deben seguir empleando los mismos métodos de planeación en la región, como fue el caso de la planeación situacional.

\section{Retos de las ciudades de América Latina y el Caribe hacia 2030}

El acelerado crecimiento de las ciudades en América Latina durante el siglo pasado ha dejado beneficios en la economía y el desarrollo, pero también ha influenciado el surgimiento de problemas que están en la agenda internacional. Hacia la primera década del siglo XXI la población de ALC rebasó los 600 millones de habitantes, más de $80 \%$ vive en ciudades; casi la mitad vive en localidades de más de un millón de habitantes, cifra superior al $36 \%$ del mundo (ONU-Habitat, 2012). Hacia 
el año 2030 , más de $80 \%$ de la población mundial vivirá en ciudades que crecerán a tasas promedio anual de entre 3 y 4\% (ONU-Habitat, 2012), en ALC la población urbana superará $90 \%$; adicionalmente, existirá una reorganización espacial de la población que llevará al surgimiento de 137 nuevas ciudades que se incorporarán al grupo de las 600 urbes más importantes del mundo (Bitar, 2014).

En este escenario ¿Cuáles serían los principales retos de las ciudades en ALC? y ¿Cómo la planeación urbana puede enfrentarlos para cumplir con los propósitos de las agendas globales? Según el estudio LAC2025 del Banco Interamericano de Desarrollo (BID, 2013), las ciudades de Bogotá y Lima se sumarán a las ciudades con más de 10 millones de habitantes, entre las que destacan la Ciudad de Méjico, Sao Paulo, Río de Janeiro y Buenos Aires; de esta manera se consolidarán tres megacorredores urbanos de importancia regional que concentrarán $20 \%$ de la población urbana de ALC: Río de Janeiro-Sao Paulo-Campinas; Toluca-Méjico- Puebla; y Buenos Aires-Rosario-Córdoba.

$\mathrm{Si}$ bien es cierto que estas concentraciones urbanas representarán una nueva dimensión del problema urbano al considerarse, tal vez, no solo como megaciudades, sino como "gigaciudades", también es cierto que incrementarán la concentración de la producción nacional y podrían ampliar las desigualdades a nivel regional, manifestando "megadiferencias". No todas las ciudades crecerán a la par, en el caso de Méjico no existen ciudades de entre 5 y 10 millones de habitantes, por ello podría intensificarse la primacía de la Ciudad de Méjico (ver gráfica 1); en el caso de Brasil también Sao Pablo observará amplias desigualdades internas, mientras que Buenos Aires podría continuar con sus grandes transformaciones.

Gráfica 1: Porcentaje de población por tamaño de ciudad

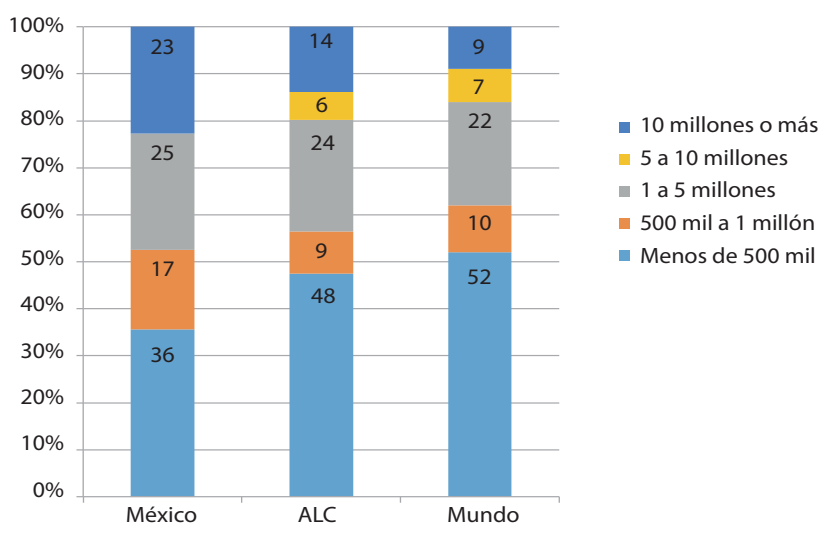

Fuente: Elaboración propia con datos (ONU-HABITAT, 2012) 
El BID ha identificado para ALC más de 140 ciudades emergentes con menos de 2 millones de habitantes que podrían duplicar su respectiva población en los próximos 20 años; sin embargo, seguirá prevaleciendo la inequidad, ya que podrían existir 160 millones de personas viviendo en condiciones precarias.

Ante este dinámico y en algunos casos controversial escenario, es importante identificar las macrotendencias con la finalidad de aprovecharlas para la generación de beneficios derivados de la urbanización, a partir de un modelo de planeación diferente o adecuado a estos nuevos retos.

\subsection{Macrotendencias que afectan la ciudad y su planeación}

\section{a) La transición demográfica}

Una de las principales características y preocupaciones en ALC es el proceso de envejecimiento de su población. La esperanza de vida al nacer a finales del siglo pasado era de 65 años y aumentó a 70 años para la segunda década del presente siglo (ONU, 2014); la tasa global de fecundidad disminuyó a lo largo del siglo pasado de seis hijos por mujer a tres para esta década, y el crecimiento de la población descendió de 2.7 a 1.6 por ciento medio anual; este comportamiento indica que la región se encuentra en una etapa avanzada de transición demográfica (Chackiel, 2004).

En materia de planeación urbana, estos cambios requerirán atención a las necesidades y relaciones de los grupos de edad avanzada principalmente -ya que en un futuro conformarán el principal grupo de edad en las ciudadesasí como de un ajuste en la dotación de equipamientos, espacios públicos e infraestructura, básicamente. Por ejemplo, la accesibilidad y emotividad para adultos mayores se han convertido en criterios de planeación y diseño urbano de los espacios públicos en ciudades que actúan anticipadamente.

El espacio público tiene la función urbana de ser un lugar de interacción por las características, necesidades y funciones sociales que se practican en él (recreación, cultura, deporte, convivencia). En algunos países existen parques orientados a personas de la tercera edad, en China, por ejemplo, se han diseñado parques públicos geriátricos o circuitos biosaludables, que son espacios verdes con mobiliario urbano de uso exclusivo.

Así, el cambio en el perfil demográfico y la interacción de los grupos de edad con el espacio establece diferentes relaciones, de tal manera que surge la necesidad de un estudio multidisciplinar entre la demografía, la psicología social, la antropología y la sociología urbana para el análisis de las relaciones espaciales, los procesos, las actividades y las personas. 


\section{b) La hiperconectividad y las nuevas formas de integración}

El uso intensivo de las TIC ha mejorado la comunicación de las personas y las distancias se han acortado; sin embargo, por contradictorio que parezca, las personas se han distanciado en el aspecto emotivo, en los espacios físicos y, en general, en sus relaciones sociales en la ciudad. La telefonía celular tiene actualmente altos niveles de penetración en casi todos los países de la región y para 2025 la banda ancha móvil podría modificar la gestión urbana, habrá una diversidad de objetos, desde aplicaciones para la movilidad hasta nuevos y más sofisticados dispositivos en autos, bicicletas y personales conectados a la red mundial, el llamado "Internet de las Cosas" (BID, 2013), los automóviles contarán con GPS y la movilidad inteligente será más común, se requerirá infraestructura para la hiperconectividad.

La hiperconectividad en la ciudad representa la producción de información a grandes escalas, es el BigDATA de la ciudad a través de redes para mejorar su administración, por ejemplo, la seguridad ciudadana, la movilidad, el medio ambiente, la gobernabilidad o la garantía de los derechos como la salud, la educación, la vivienda y los servicios. De esta manera, existirá tal nivel de flujo de información que será necesario dotar infraestructura, equipamiento y capacidad de procesamiento para aprovechar el beneficio de los flujos de información.

Las ciudades requieren nuevas formas de relacionarse, basadas en el uso de las TIC; por otro lado, la falta de infraestructura y conectividad podría ser considerada una nueva forma de marginación o segregación, tal es el caso del analfabetismo digital, la brecha digital o el desigual acceso a información y conocimiento.

\section{c) La movilidad}

La cantidad de horas que actualmente pasan las personas en los traslados para realizar sus actividades en la ciudad se ha convertido en un reto. Para 2025 las ciudades de América Latina tendrán alrededor de 140 millones de automóviles, 80 millones más que en 2010 (BID, 2013), con la hiperconectividad se podrá monitorear a distancia el flujo de tráfico y anticipar puntos de congestión "en tiempo real".

Además, más ciudades contarán con Buses de Tránsito Rápido (BRT), se construirán nuevos sistemas de transporte subterráneo (Metro) y aumentará el uso de la bicicleta como alternativa de transporte (BID, 2013). Adicionalmente, continuarán nuevas formas de movilidad como es el caso de "uberópolis" o los "ridehailing". Uber ha realizado más de 2000 millones de viajes en 400 ciudades del mundo desde el inicio de sus servicios en América Latina (Kostenwein, 2017). 
En 2013 el informe de la ONU sobre los asentamientos humanos detalló estrategias de movilidad urbana sostenible, haciendo énfasis en una forma diferente de analizarla y atenderla. La movilidad implica flujos de personas y bienes, pero, sobre todo, es un asunto de facilidad para que las personas realicen sus actividades, accedan a ellas de forma eficiente, incluso sin moverse del lugar. Para facilitar la accesibilidad se requiere impulsar áreas de uso mixto, implementar sistemas intermodales que disminuyan el uso del automóvil y usar de forma inteligente las TIC.

Si bien este reto podría representar uno de los principales en la ciudad, tal vez implica un cambio radical en la forma como se resuelve el problema, es decir, pasar de enfocar los problemas de movilidad desde cómo mover más gente en menos tiempo hasta cómo evitar que la gente se mueva.

\subsection{Los retos y la nueva agenda urbana ALC2030}

La transición demográfica por la que atraviesan y atravesarán las ciudades de la región debe considerar la forma de articular la planificación y el diseño urbano, y la generación y adecuación de espacios flexibles que se ajusten al proceso de cambio de actividades de las personas y a su composición demográfica.

La planeación y el diseño urbano debe basarse en el reconocimiento al uso intensivo de las TIC en las ciudades, que han marcado nuevas formas de relación y de integración del espacio urbano, por ello deben ser empleadas en mejorar la integración bajo el enfoque tradicional de comunidad, de sociedad red y para hacer eficiente la administración de la ciudad, a partir del uso de información.

La movilidad, sin lugar a dudas, debe ser "el problema a resolver", ya que representa el común denominador en todas las ciudades y condiciona la productividad, la calidad de vida, la seguridad y muchos otros elementos de interacción que caracterizan la ciudad. En caso de no atender el problema de la movilidad, las macrotendencias pueden convertirse en riesgos.

En este orden de ideas, los ODS deben orientar la responsabilidad y el compromiso de cada acción urbana, la prosperidad como el enfoque que permita integrar el sector productivo con la calidad de vida de las personas, considerando elementos económicos, sociales, ambientales, tecnológicos, de gobierno y de infraestructura medibles que, en el marco de cada realidad local, permita identificar una ruta para alcanzar un mejor estado.

La gobernabilidad debe considerar, además de lo tradicional, la forma de enfrentar los retos, cómo se utilicen las tecnologías, generar movilidad 
inteligente y establecer nuevas relaciones. Un gobierno urbano tendrá éxito político y técnico en la medida que logre enfrentar y aprovechar estas situaciones a través de un diseño que agregue valor al ciudadano, articulado con una efectiva planeación urbana que mejore la percepción que las personas tienen sobre su espacio urbano.

Existen condiciones que afectan la vida de las personas y modifican su percepción, estado de ánimo y hasta la preferencia por vivir en un lugar, tal es el caso de la imagen urbana, el impacto de la obra pública, la calidad de la vivienda, la movilidad, el diseño de los espacios públicos, el nivel de empleo, la seguridad, la identidad social, el medio ambiente, la salud, la educación, entre muchos otros. Bajo esta lógica es necesario lograr una urbanización de calidad (Jordan \& Simioni, 2002), una urbanización que genere valor.

La Nueva Agenda Urbana, impulsada por la Organización de las Naciones Unidas, centra la preocupación en las personas y sus relaciones, en la planeación y el diseño urbano como herramientas de cambio, basadas en instrumentos legales, institucionales y financieros. Reconoce la importancia que tiene la ciudad en el desarrollo global de las naciones $y$, por ello, resalta los beneficios de la urbanización; sin embargo, advierte también sobre las consecuencias de un crecimiento urbano desmedido, descontrolado y con ausencia de derechos humanos.

Por su parte, el PAR para América Latina representa el vínculo bajo el cual los países y sus ciudades habrán de coordinar acciones hacia un propósito global definido en el Objetivo 11 de Desarrollo Sostenible, pero ¿cuáles habrán de ser los procesos y métodos bajo los cuales se aplicará dicho plan? La Organización de las Naciones Unidas, a través de la Comisión para América Latina y el Caribe (CEPAL), impulsó durante décadas procesos de planeación situacional que implicaban diagnósticos detallados sobre los problemas del desarrollo, apropiados en la planeación urbana; posteriormente, la influencia de otras corrientes como la planeación estratégica y la prospectiva representó métodos para realizar planes, estudios y proyectos urbanos, ¿qué seguirá con la NAU para enfrentar los retos en las ciudades?

A partir del reconocimiento de las macrotendencias, es fundamental orientar la discusión del desarrollo de las ciudades hacia un método de planeación centrado en la persona, en la garantía de sus derechos humanos y urbanos, en la dualidad global desde lo local. De esta manera será necesario integrar escalas y formas de relacionarse en la ciudad, desde el barrio hasta lo internacional, incorporando a la ciudad en la red de ciudades globales. 


\section{La escala global y la necesidad de pensar en las personas}

Los grandes cambios y ajustes de los modelos económicos en las diferentes regiones del mundo han generado transformaciones y nuevas formas espaciales que, a su vez, han cambiado la conformación de las ciudades, a tal grado que se considera una revolución de la urbanización (González, 2012; Iracheta, 2005). La globalización vincula prácticas locales de convivencia con relaciones sociales a nivel mundial y reorganiza aspectos de la vida cotidiana; en este contexto las ciudades han cobrado mayor importancia, entre otras cosas, por funcionar como nodos articuladores, ya que ocupan un lugar en el mundo por la red de ciudades a la que pertenecen.

Sassen (1995) afirma que la influencia de la globalización y sus procesos económicos, políticos, sociales y culturales ha generado nuevos espacios de multirrelaciones, como la ciudad global. Esta ciudad es un núcleo que concentra capital económico, humano, gestiona el conocimiento de manera estratégica y agrega valor a su región. Su importancia económica, flujos de información, mercancías y personas van más allá de sus espacios físicos, alcanzando una influencia internacional. Como parte de un sistema transnacional, la ciudad global genera conocimiento y servicios especializados, forma parte de una economía de agregación y por ello depende cada vez menos del territorio contiguo para la generación del capital (Sassen, 1995).

A.T. Kearney ${ }^{1}$ publica cada dos años desde 2008, a través de Foreign Policy ${ }^{2}$, el Ranking de Ciudades Globales, considerando 84 ciudades del mundo evaluadas mediante 26 criterios agrupados en cinco rubros: Actividad empresarial, Capital humano, Intercambio de información, Experiencia cultural e Influencia en la política.

New York es la ciudad global número uno (Ver tabla 1), Londres es el segundo lugar, le siguen París, Tokio y Hong Kong. De las 20 primeras ciudades, siete son de la región Asia Pacífico (Tokio, Hong Kong, Beijing, Singapur, Seúl, Sídney y Shanghái), otras siete de Europa (Londres, París, Bruselas, Madrid, Viena, Moscú y Berlín), y seis de América (Nueva York, Los Ángeles, Chicago, Washington, Toronto y Buenos Aires).

1 A.T. Kearney es un equipo de consultores de influencia internacional; desde 1926 ha realizado estudios y este índice de ciudades globales lo realiza cada dos años desde 2008.

2 Para mayor referencia ver http://www.fp-es.org/ranking-de-ciudadesglobales-2010 
Tabla 1: Índice de las principales 20 Ciudades Globales

\begin{tabular}{|l|c|c|c|c|}
\hline \multicolumn{1}{|c|}{ Ciudad Global } & $\mathbf{2 0 1 4}$ & $\mathbf{2 0 1 2}$ & $\mathbf{2 0 1 0}$ & $\mathbf{2 0 0 8}$ \\
\hline Nueva York & 1 & 1 & 1 & 1 \\
Londres & 2 & 2 & 2 & 2 \\
\hline París & 3 & 3 & 4 & 3 \\
Tokio & 4 & 4 & 3 & 4 \\
Hong Kong & 5 & 5 & 5 & 5 \\
Los Ángeles & 6 & 6 & 7 & 6 \\
Chicago & 7 & 7 & 6 & 8 \\
Beijing & 8 & 14 & 15 & 12 \\
Singapur & 9 & 11 & 8 & 7 \\
Washington & 10 & 10 & 13 & 11 \\
Bruselas & 11 & 9 & 11 & 13 \\
Seúl & 12 & 8 & 10 & 9 \\
Toronto & 13 & 16 & 14 & 10 \\
Sídney & 14 & 12 & 9 & 16 \\
Madrid & 15 & 18 & 17 & 14 \\
Viena & 16 & 13 & 18 & 18 \\
Moscú & 17 & 19 & 25 & 19 \\
Shanghai & 18 & 21 & 21 & 20 \\
Berlín & 19 & 20 & 16 & 17 \\
Buenos Aires & 20 & 22 & 22 & 33 \\
\hline
\end{tabular}

Fuente: A.T. Kearney Global Cities Index y Emerging Cities Outlook 2016.

Para insertar a una ciudad de forma exitosa en la escala mundial es necesario incrementar el talento humano, desarrollar sistemas de información "robustos", el BigDATA de la ciudad, que sus ciudadanos estén conectados a través de las TIC, que sus espacios culturales se consoliden como patrimonio a nivel internacional (que generen valor), y sobre todo que la percepción al exterior esté basada en una importante estrategia de comunicación, es decir, se consolide "una marca ciudad" para llevar lo local a nivel global.

Sassen (1999) subraya que es necesario incrementar los servicios especializados para mejorar la organización de la ciudad y como base para el desarrollo de nuevas actividades económicas. Los servicios de alto valor agregado, el conocimiento y el perfil de los profesionales orientado a las tecnologías se han convertido, tal vez, en los principales activos para 
las nuevas funciones de la ciudad. De esta manera las actividades son generadoras de valor en la ciudad.

El análisis urbano es altamente complejo debido a las relaciones de escala global, pero sobre todo como resultado de la hiperconectividad, de las mercancías, del capital y de las personas. Por lo anterior, es importante contar con proyectos urbanos que conserven, restauren o generen capital; una ciudad que trabaja en ello se vuelve más competitiva, genera ingresos para sus habitantes, actividades, servicios y activa la economía en general; los capitales son también los elementos naturales y forman parte de un enfoque más sustentable en la ciudad.

Las ciudades en sí mismas concentran capitales, aunque es necesaria una visión de largo plazo y una efectiva gestión para detonar actividades a cualquier hora del día. Casi todas las ciudades tienen horarios establecidos que regularmente se ajustan a la actividad laboral, sin embargo, pocas administran la actividad turística y cultural en horarios nocturnos para "vivir la ciudad de noche". Ciudades como Londres en Inglaterra o Melbourne en Australia han desarrollado políticas urbanas centradas en la mejora de la calidad de los espacios a partir del comportamiento de las personas en horario nocturno, haciendo énfasis en la diversidad de uso y en las ventajas económicas.

Algunos ejemplos que en América Latina han logrado consolidarse por sus actividades nocturnas en beneficio de la sociedad son (BID, 2016): Buenos Aires, que organiza "La Noche de los Museos"; Caracas, Venezuela, que organiza el festival "Por el Medio de la Calle"; Asunción, Paraguay, con su centro histórico y sus actividades comerciales. El Banco Interamericano de Desarrollo (BID) publicó las ventajas de promover una economía nocturna en las ciudades (empleo, identidad, mejora de la imagen urbana, aprovechamiento del espacio público) en el marco de la Iniciativa de Ciudades Emergentes y Sostenibles. Bajo esta iniciativa el reto de la ciudad es relacionar de forma productiva las actividades económicas y el medio ambiente, a través de la sostenibilidad urbana y un crecimiento urbano ordenado.

De esta manera, la importancia de desarrollar actividades en la ciudad que generen valor centra la discusión de orden global en las personas y sus relaciones en la ciudad, también recupera la comunicación y la identidad de los espacios como los elementos estratégicos para el desarrollo de una ciudad a nivel global. La conectividad tanto física como virtual se convierte en la premisa fundamental para integrar los espacios, comunicarlos y relacionarlos. 


\subsection{El crecimiento urbano articulado}

Actualmente existe un debate sobre el modelo de ciudad ¿Debemos seguir con las ciudades extensas y dispersas o bien invertir en ciudades compactas? La discusión sopesa los impactos tanto positivos como negativos de ambos modelos y evalúan cuál conviene más en ALC; lo que evidencia la discusión es que una ciudad mal planificada sí es más costosa, ¿cuáles son los principales aportes de cada modelo?

La idea de "ciudad compacta" no implica un crecimiento al interior, sino el aprovechamiento de los espacios, por tanto, se desarrolla no solo con construcciones verticales, sino con la reutilización y aprovechamiento de espacios subutilizados. El Banco Interamericano de Desarrollo (BID) estima que para mediados del presente siglo las ciudades en los países en desarrollo duplicarán su población y triplicarán la superficie de las manchas urbanas actuales, es decir, el patrón de ciudad extensa podría continuar.

A partir de esta preocupación, el BID hace cuatro recomendaciones para la planeación de ciudades compactas, con la idea de aprovechar los beneficios y disminuir los efectos negativos de este patrón de crecimiento: disponibilidad de uso de suelo, revisión de los derechos de propiedad y de desarrollo inmobiliario, nuevos esquemas de articulación, alianzas y acuerdos institucionales, y sistemas de inteligencia urbana.

Sobre las ciudades que crecen de forma extensa, basan su desarrollo en modelos de transporte que conecten a la ciudad de forma funcional a través de espacios que mejoran y dignifican la calidad de vida de las personas. Si bien este modelo es altamente cuestionable, en casos como Norteamérica, Alemania o algunos países de América Latina ha permitido mejorar condiciones de reproducción, inclusión y menores costos en la "periferia de la ciudad".

Existen ventajas y desventajas de ambos modos de crecimiento, entonces un crecimiento urbano ordenado inclusivo tendría que recuperar el "derecho" de cada ciudadano por construir su espacio, generando sus propias ventajas de acceso a una mejor calidad de vida. Por ello, más que la imposición de un modo de crecimiento opuesto a la tendencia que ha observado cada ciudad, debería de ser un modo de crecimiento centrado en las personas y su derecho a construir su propio espacio, un modo de crecimiento que genere valor y obtenga beneficios de la urbanización.

\subsection{El derecho a la ciudad}

La NAU recupera el Derecho a la Ciudad, orientado a los derechos humanos que deben estar garantizados en ella; pero también es necesario considerar 
que los nuevos problemas y retos que enfrenta la ciudad implican el surgimiento y necesidad de garantizar nuevos derechos urbanos.

El derecho enfocado al ámbito urbano reivindica la garantía de nuevas relaciones de respeto y obligaciones para y con los ciudadanos. En septiembre de 2005 se elaboró la Carta Mundial del Derecho a la Ciudad como un acuerdo de la ONU y un llamado de atención sobre la importancia de ampliar el enfoque tradicional sobre la mejor calidad de vida de las personas, desde la vivienda y el barrio hasta la ciudad y el entorno rural de influencia. De esta manera, el Derecho a la Ciudad surge como un concepto que busca revindicar la forma como se está "construyendo" la ciudad y enfatizar una nueva manera de promoción, respeto, defensa y realización de los derechos humanos fundamentales, los derechos civiles, políticos, económicos, sociales, culturales y ambientales garantizados en los marcos jurídicos internacionales, nacionales y regionales.

Este concepto no es nuevo, surge en la Sociología urbana francesa, pero actualmente ha cobrado importancia como parte del discurso de políticos, organizaciones sociales y en algunos casos ya forma parte de la política urbana. En 2016 la ONU lo incluyó en la Nueva Agenda Urbana, gobiernos nacionales lo han llevado a su propia Constitución o leyes nacionales como el caso de Méjico; sin embargo, no en todos los casos se ha manejado la precisión conceptual, de instituciones para su garantía, o límites espaciales para determinar funciones, atribuciones o responsabilidades de las autoridades.

Académicos han cuestionado su existencia para explicar la idea como "los derechos en la ciudad", más que "el derecho a la ciudad"; es decir, reconocer la existencia de derechos como la educación de calidad, la vivienda digna, los servicios, o incluso el acceso a internet, entre muchos otros; así, tal vez, los derechos en la ciudad podrían ser garantizados. Ahora es tiempo de reflexionar sobre la pertinencia del derecho a la ciudad en el uso público en beneficio de las personas.

Con los retos actuales existen nuevos derechos, por ejemplo, el derecho a una movilidad activa. Ya que las ciudades están construidas para el automóvil, representa más que el derecho al libre tránsito, implica la garantía de contar con infraestructura para desplazarse con seguridad, caminando o en bicicleta. En este sentido, el derecho a la ciudad implica el reconocimiento y garantía de orden, seguridad e inclusión de las personas en el espacio urbano, como resultado de los nuevos retos y problemas que enfrentamos.

\subsection{Las personas al centro del diseño y la planeación urbana}

Como elemento de calidad de vida, el diseño del entorno influye en el 
estado emocional de las personas, en su percepción o identidad; es una actividad que como parte de un proceso genera valor.

El Índice de Calidad de Vida en la ciudad, elaborado por Mercer, evaluó 440 ciudades de todo el mundo mediante 39 variables agrupadas en 10 categorías, donde Viena es la mejor ciudad en Calidad de Vida por dos años consecutivos. La ciudad de América Latina mejor posicionada en este ranking es Montevideo (lugar 78), seguida de Buenos Aires (91) y Santiago (93); la Ciudad de Méjico se ubica en el lugar 126, perdiendo 4 posiciones respecto de 2013.

La revista Forbes publicó en su edición 2016 el ranking de las 10 mejores ciudades para vivir. Las tres primeras posiciones corresponden a Viena (Austria), Zúrich (Suiza) y Auckland (Nueva Zelanda), principalmente porque cuentan con un buen entorno económico, social y de seguridad. Este índice enlista 230 ciudades de todo el mundo y se basa en 39 factores agrupados en 10 categorías.

¿Podría ser que las ciudades de Europa consoliden su orientación de planeación urbana hacia la persona, para ser las mejores posicionadas en este ranking? La planeación a nivel local y las variables económicas del entorno nacional son elementos clave en Austria, Suiza y Nueva Zelanda; la calidad de vida está determinada principalmente por niveles altos de empleo e ingreso, pero también de aspectos locales como seguridad personal, compromiso cívico, educación y relación vida-trabajo, de tal manera que existe una mezcla de buenas situaciones (entorno favorable) para el desarrollo de las personas.

Existen compañías multinacionales que dedican recursos compensatorios para mejorar el nivel de vida de sus trabajadores. Bajo la premisa que el desempeño es mayor en personas con mejor calidad de vida, existen dos incentivos: la calidad de vida y la prima por movilidad, estas compensaciones cubren los costos generados por la ciudad para que la persona -en este caso el trabajador- no se vea afectado.

La planeación urbana en estas ciudades que buscan mejorar la calidad de vida centra sus políticas en el diseño, la percepción y la inclusión de las personas en el espacio urbano, así como en su economía. La infraestructura cumple entonces con una función de utilidad y diseño, por ejemplo, permite tanto la conectividad física como virtual, pero también actúa como elemento de infraestructura del paisaje. La accesibilidad como criterio de planeación deberá buscar la inclusión, considerando que no solo se trata de la disposición o adecuación del espacio físico o de las garantías para grupos en desventaja, sino también de generar espacios que permitan "estar en el lugar", independiente de su condición física, 
económica o social. Finalmente, la generación o conservación de valor en ciudades permitirá generar capitales o mantener los existentes, como es el caso de los espacios culturales, históricos, patrimoniales o a las nuevas vocaciones científicas y económicas que hacen destacar a una ciudad en el contexto internacional (Alva \& Narváez, 2015).

A partir de lo anterior, podría generarse una forma diferente de hacer planeación bajo la premisa de la centralidad en la persona, que integre los principales elementos discutidos: la persona, sus actividades, los procesos, las relaciones, así como la accesibilidad, la inclusión, la conectividad, la integración y la generación de valor.

\section{Hacia una agenda de planeación urbana centrada en la persona}

En el contexto internacional, hacia 2030 podría existir una reestructuración tanto de las actividades como de las grandes ciudades, al menos eso podría suceder en 200 de las 600 ciudades más pobladas; las ciudades que superen con éxito dicha reestructuración será porque logren resolver los problemas de movilidad, sustentabilidad ambiental, energía, comunicación digital, gobernabilidad y educación (BID, 2013).

La CEPAL (2003) señala que las ciudades de América Latina enfrentan también otros desafíos como la falta de planificación, debilidad institucional, carencia de espacios públicos, segregación, exclusión, desigualdad, inseguridad y crecimiento exponencial del parque automotriz, lo cual representa una desventaja para insertarse en la economía mundial. En este sentido, parece que los gobiernos, sobre todo locales, deberían intervenir estratégicamente en áreas de alto valor agregado para la innovación, mejorar la competitividad y sustentabilidad urbana. Para ello se requiere una ambiciosa reforma urbana, sobre todo institucional, es decir, reingeniería del gobierno local que aliente la inversión y genere nuevas formas de "hacer ciudad". En este escenario la persona deberá estar en el centro de la política urbana.

Con la finalidad de integrar todos los elementos discutidos en los apartados anteriores, así como las premisas de la NAU y el PAR, se propone un modelo de planeación urbana centrado en la persona y basado en tres conceptos clave: la conectividad para lograr una mejor integración, la accesibilidad para garantizar la inclusión y la generación de valor para fortalecer la identidad y el patrimonio urbano.

\subsection{Conectividad}

La conectividad implica la posibilidad de que personas y espacios estén articulados a través de una amplia red de infraestructura que permita 
al ciudadano movilizarse entre los principales puntos y llegar en corto tiempo a realizar sus actividades, así como integrar grandes espacios a través de recorridos histórico-culturales, turísticos o con una función económica específica.

La planificación y el diseño urbano es la principal herramienta de los gobiernos locales para el desarrollo de infraestructura que permita integrar mejor los territorios, agregar valor y centrarse en el equilibrio entre la persona y el medio ambiente, sobre todo con visión de largo plazo.

Es importante subrayar que esta forma de planeación implica la generación de nuevas alianzas, acuerdos y responsabilidades entre el sector público, urbanistas, desarrolladores inmobiliarios, empresas y la sociedad organizada; las sinergias y redes de colaboración -gobierno, academia, empresa, sociedad- podrían ser el principal instrumento para financiar los grandes proyectos de infraestructura como factor de integración y con perspectiva global.

\subsection{Accesibilidad}

El diseño urbano universal parece que aún no se convierte en un tema de política urbana en ALC, solo destacan algunas ciudades como Curitiba. Aunque tradicionalmente se ha considerado la inclusión en la ciudad como resultado de la accesibilidad al espacio físico y para las personas con capacidades diferentes, actualmente la concepción de accesibilidad e inclusión se ha ampliado.

La accesibilidad se refiere, primero, a disponer o no de un espacio; segundo, a la adecuación del espacio para determinadas condiciones; tercero, a la disponibilidad de servicios virtuales como el acceso a la banda ancha; cuarto, a la posibilidad o no de poder habitar en un determinado espacio independiente de la capacidad económica de las personas $y$, finalmente, a la emotividad, pertenencia y apropiación del espacio urbano, de tal suerte que es necesario considerar estas cinco dimensiones para logar una ciudad incluyente.

La accesibilidad representa una de las principales garantías en el derecho a la ciudad por su misma naturaleza social, con un enfoque no solo físico, sino también intangible, por ello se valora con el propósito de la inclusión.

\subsection{Generación de valor}

Pensar en el diseño de una ciudad implica un proceso que, como en otras disciplinas, parte de un proceso y una cadena de valor, en este caso integrada por los diferentes actores de la ciudad. Bajo esta perspectiva 
se recuperarían y evaluarían los beneficios de la urbanización, no solo a partir de economías de escala o mejora en la calidad de vida, sino en la generación de valor como resultado de la integración de actividades.

Con la infraestructura del paisaje se podrían generar proyectos de gran escala que generen valor en la ciudad, que representen un activo para las actividades artísticas, culturales o turísticas, además, como espacios públicos para la convivencia. De esta manera sería posible conectar a la ciudad a través de diferentes puntos que sean un referente para ella.

En síntesis, la planeación centrada en la persona tendría como fin diseñar un proyecto de ciudad a partir de la construcción de nuevos acuerdos, el reconocimiento de nuevos retos y la implementación de tres elementos que den certeza a la sociedad, orden al crecimiento urbano, modernización de la administración del espacio urbano y certidumbre en el futuro. Así, la conectividad, accesibilidad y el patrimonio representan atributos que giran en torno al ciudadano y lo colocan en el centro de la planeación urbana para el diseño de una ciudad que agrega valor.

\subsection{Reflexiones finales}

La región de América Latina y el Caribe requiere de nuevos modelos de planeación, que por un lado permitan implementar la Nueva Agenda Urbana, pero que por otro inserten a las ciudades con ventaja en la dinámica global, bajo un enfoque de integración y centrado en la persona desde lo local.

El enfoque de integración y de un nuevo modelo de planeación urbana es necesario ante las nuevas disyuntivas que plantea la economía mundial y la reorganización de los modelos de crecimiento, pero sobre todo por el ampliamente discutido y cuestionado papel de la urbanización y sus ventajas para el desarrollo. Las ciudades de ALC requieren posicionarse a escala mundial como una red de ciudades fortalecidas, que permita generar nuevos liderazgos en la economía mundial altamente competitiva.

Por su parte, un modelo urbano que logre articular a la planeación con el diseño urbano será un activo para avanzar hacia la construcción de ciudades más humanas, sostenibles y prósperas. Por ello, el desarrollo de infraestructura para la conectividad desde la escala individual hasta la escala global es una exigencia que podría convertirse en derecho y no solo una necesidad; la inclusión para generar mejores condiciones de equilibrio y disminuir las desigualdades es ya una responsabilidad compartida y un reto para la ciudad; y la generación de valor constituye una nueva forma de activos para que las ciudades se posicionen $y$, sobre todo, mejoren la calidad de vida de las personas. 
ALC debe transitar hacia 2030 con un avance en el logro de los ODS, no solo en algunas ciudades, sino como región para poder enfrentar, además, las grandes desventajas generadas por las economías del norte de América, de Europa y Asia. Solo con nuevos modelos de desarrollo y metodologías se podrán construir nuevas ciudades en medio de nuevos retos globales.

En este documento se ha hecho una síntesis de los principales resultados de informes de organismos internacionales, con la finalidad de rescatar y unificarlos en macrotendencias urbanas. A partir de ellas se rescataron los principales puntos que dan forma a un modelo de planeación que busca centrar su preocupación en la persona, sus relaciones, actividades y procesos en la ciudad. El modelo, además de la parte analítica expuesta en este artículo, se basa en los postulados de la sociología urbana francesa, el nuevo urbanismo y el urbanismo del paisaje, así como en los trabajos de Jan Ghel sobre ciudades para las personas y protocolos de diseño urbano de ciudades australianas, "creando lugares para la gente".

\section{Referencias Bibliográficas}

1. ALVA, Benjamín, \& NARVÁEZ, Fernando. (2015). Diseño centrado en la persona, Tendencias y Retos para los espacios urbanos. En: ASINEA93, Innovación educativa para la arquitectura: Compromiso de enseñanza aprendizaje. Méjico: ASINEA.

2. BID. (2013). LAC 2025, América Latina y el Caribe en 2025. Obtenido de: Banco Interamericano de Desarrollo. Disponible en internet: http://www.iadb.org/es/ videos/ver,2173.html?videoid=10103\#.Vys1PISLSCg. Consultado 11.11.2016

3. BID. (2016). La ciudad de noche. Disponible en internet: https://blogs.iadb.org/ sinmiedos/2016/09/15/video-ciudades-nocturnas/. Consultado el 17.01.2017

4. BITAR, Sergio. (2014). Las tendencias mundiales y el futuro de América Latina. En: Serie Gestión Pública, Serie Gestión Pública N 78, CEPAL, CEPAL. Santiago de Chile: Naciones Unidas.

5. CEPAL. (2003). Elementos clave y perspectivas prácticas en la gestión urbana. Santiago de Chile: Comisión Económica para América Latina y el Caribe, Organización de las Naciones Unidas.

6. CHACKIEL, Juan. (2004). La dinámica demográfica en América Latina. En: Serie Población y Desarrollo. Centro Latinoamericano y Caribeño de Demografía (CELADE) - División de Población. Santiago de Chile: Naciones Unidas.

7. GONZÁLEZ, Sergio (2012). Panorama del Desarrollo Territorial en América Latina y el Caribe, Santiago de Chile, ILPES-CEPAL-ONU-GIZ. Pp.142 
8. IRACHETA, Alfonso, (2005). Las revoluciones que transforman el mundo. En Revista Bitácora Urbano Territorial, Vol 9. Bogotá: Universidad Nacional de Colombia. Pp. 54-63

9. JAN, Gehl. (2014). Ciudades para la gente. Buenos Aires: Infinito.

10. JORDAN, Ricardo \& SIMIONI, Daniela. (2002). Hacia una nueva modalidad de gestión urbana. En: CEPAL, las nuevas funciones urbanas: gestión para la ciudad sostenible. Santiago de Chile: CEPAL.

11. KOSTENWEIN, David. (2017). Uberópolis: los efectos del ridehailing en nuestras ciudades. Obtenido de Ciudades Sostenibles. Disponible en internet: http:// blogs.iadb.org/ ciudadessostenibles/2017/01/18/ridehailing/. Consultado el 15.02.2017

12. KRUGMAN, Paul. (2004). El internacionalismo moderno. Barcelona: Planeta de Libros-Crítica.

13. ONU. (2005). Carta mundial por el derecho a la ciudad. Barcelona: Organización de las Naciones Unidas.

14. ONU. (2014). La situación demográfica en el mundo en 2014. Disponible en internet: http://www.un.org/en/development/desa/population/events/pdf/ other/4/World\%20Population\%20Situation_2014_10\%20key\%20findings_ es.pdf. Consultado el 01.18.2017

15. ONU-Hábitat. (2012). Estado de las Ciudades de América Latina y el Caribe. Brasil: Hábitat publications.

16. ONU-Hábitat. (2012). Estado de las ciudades en América Latina y el Caribe 2012. Rumbo a una nueva transición urbana. Nairobi: Programa de las Naciones Unidas para los Asentamientos Humanos ONU-Hábitat.

17. ONU-Hábitat. (2013). Iniciativa para la prosperidad de las ciudades. Organización de las Naciones Unidas.

18. SASSEN, Saskia. (1995). La ciudad global: "Una introducción al concepto y su historia". En: S. Sassen, La ciudad global. Rhode Island: Brown Journal of World Affairs.

19. SASSEN, Saskia. (1999). La ciudad global: Nueva York, Londres, Tokio, Buenos Aires. Nueva Jersey: Eudeba.

20. STIGLITZ, Joseph. (2015). El Malestar de la globalización. Madrid: De Bolsillo. 
Parece mentira que la ciudad que se extiende a los pies del mítico Sumaq Urqu o Cerro Rico (4782 msnm) haya sido en el siglo $X V I I$, una de las más grandes del mundo. Por entonces, y con 160.000 habitantes, la Villa Imperial de Potosí era más grande que Londres, y por supuesto más rica que la urbe británica. El pastor quechua Diego Huallpa descubrió las primeras vetas en 1545 y desde entonces los invasores españoles explotaron las minas sometiendo a cientos de miles de indígenas, que murieron esclavizados, enfermos o masacrados en las rebeliones que intentaron derribar el yugo español antes de la Independencia. Casi todo el corazón de la montaña estaba formado de plata y por ello durante casi 200 años, la mitad de toda la plata del mundo, provenía de este cerro mágico del Alto Perú. Símbolo de la enorme riqueza argentaria de Bolivia, representada en su escudo nacional, la montaña llenó de opulencia durante casi tres siglos, las arcas del imperio español, para pagar muchas guerras y todas las extravagancias de la corte. Llegado el declive, cesó la avalancha del metal por el Río de La Plata, y Potosí -otrora envidia de los reyes del mundo-se sumió en el olvido, víctima de la maldición de su propia riqueza.

Héctor José Sarmiento Politécnico Colombiano.

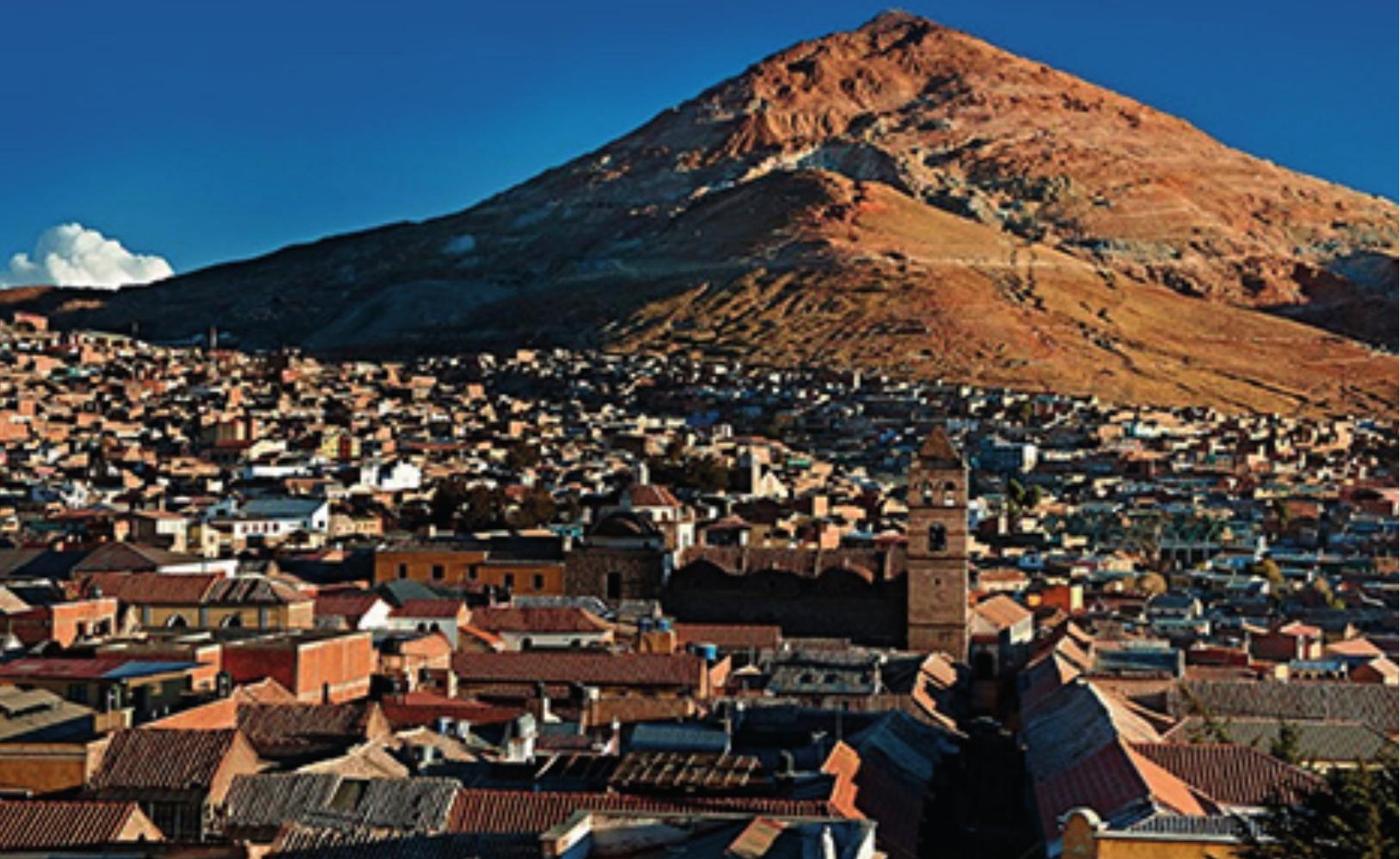

